\title{
Benefit of surgery after chemoradiotherapy in stage IIIB (T4 and/or N3) non-small cell lung cancer
}

Dominique H. Grunenwald, MDa

Fabrice André, $\mathrm{MD}^{\mathrm{b}}$

Cécile Le Péchoux, MD

Philippe Girard, MDa

Christian Lamer, MD

Agnès Laplanche, $\mathrm{MD}^{\mathrm{e}}$

Michèle Tarayre ${ }^{\mathrm{e}}$

Rodrigo Arriagada, $\mathrm{MD}^{\mathrm{c}}$

Thierry Le Chevalier, MD ${ }^{b}$
From the Thoracic Department, Institut Mutualiste Montsouris, ${ }^{\text {a Paris; }}$ the Departments of Medicine ${ }^{b}$ and Radiotherapy, Institut Gustave Roussy, Villejuif; the Intensive Care Unit, Institut Mutualiste Montsouris, ${ }^{\mathrm{d}}$ Paris; and the Department of Biostatistics, ${ }^{\text {e }}$ Institut Gustave Roussy, Villejuif, France.

Received for publication Dec 20, 2000; revisions requested Feb 27, 2001; revisions received March 27, 2001; accepted for publication April 13, 2001.

Address for reprints: D. H. Grunenwald, MD, Head, Thoracic Department, Institut Mutualiste Montsouris, 42 bld Jourdan, 75674 Paris cedex 14, France (E-mail: thorax@imm.fr).

J Thorac Cardiovasc Surg 2001;122:796-802

Copyright @ $\odot 2001$ by The American Association for Thoracic Surgery

0022-5223/2001 \$35.00+ $0 \quad \mathbf{1 2 / 1 / 1 1 6 4 7 2}$

doi:10.1067/mtc.2001.116472
Objective: The purpose of this study was to evaluate postchemoradiotherapy surgery in stage IIIB non-small cell lung cancer.

Methods: Forty patients with stage IIIB non-small cell lung cancer were included in this phase II study. A preoperative diagnosis of stage IIIB cancer was based on mediastinoscopy or a thoracotomy in all patients. Induction treatment included two cycles of cisplatin (100 mg/m $\mathrm{m}^{2}$, day 1$)$, 5 -fluorouracil ( $1 \mathrm{~g} / \mathrm{m}^{2}$, days $\left.1-3\right)$, and vinblastine (4 $\mathrm{mg} / \mathrm{m}^{2}$, day 1) combined with $42 \mathrm{~Gy}$ of hyperfractionated radiotherapy delivering 21 Gy in two sessions. Patients with a clinical response were offered surgery.

Results: The minimum follow-up for survivors was 48 months. Thirty patients had a T4 lesion and 18 had N3 disease. Twenty-nine patients (73\%) had a clinical objective tumor response after induction treatment. These 29 patients underwent thoracotomy, and a complete resection was performed in $23(58 \%)$. Two postoperative deaths occurred (7\%). Four patients had a pathologic complete response at the time of surgery (10\%). The 5-year survival is $19 \%$ for the overall population. When only patients who had persistent viable tumor cells at surgery are considered $(n=25)$, the 5-year survival is $28 \%$. The 5-year survival is $42 \%$ for patients having no mediastinal lymph node involvement at the time of surgery and being treated with complete resection.

Conclusion: This study shows that surgery, when feasible, is associated with a $28 \%$ long-term survival for patients in whom chemoradiotherapy alone fails to control disease.

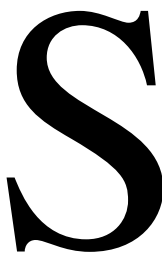

tage IIIB disease is considered inoperable and the patients are usually treated with chemoradiotherapy. ${ }^{1-3}$ An analysis of relapse sites has shown that locoregional failure is the major stumbling block in these patients ${ }^{4}$ because the 1-year locoregional control rate was only $17 \%$ for patients treated with chemoradiotherapy. In the same trial, chemotherapy dramatically decreased the rate of distant metastasis from $67 \%$ to $45 \%$. Since then, postinduction surgery has been investigated as a possible alternative means of improving locoregional control of stage IIIB disease. Postinduction surgery was reported to be feasible and complete resection possible in more than $50 \%$ of cases. Only a few studies, ${ }^{5-7}$ however, included more than 20 patients, and the median followup was short in all of them. The long-term evaluation of postinduction surgery in a large phase II study has yet to be attempted. The aim of the present phase II study was to evaluate the long-term efficacy of postinduction surgery for patients with stage IIIB 
$\underline{\text { Initial staging }}$

Induction chemoradiotherapy

\begin{tabular}{|l|l|} 
Chemotherapy: & 5 FU CP $1 \mathrm{~g} / \mathrm{m} 2 \mathrm{D} 1-\mathrm{D} 3, \mathrm{D} 31-33$ \\
& CDDP $100 \mathrm{mg} / \mathrm{m} 2 \mathrm{D} 1, \mathrm{D} 31$ \\
& Vinblastine $4 \mathrm{mg} / \mathrm{m} 2 \mathrm{D} 1, \mathrm{D} 31$ \\
Chest radiotherapy: & 21 gy $(1.5 \mathrm{~Gy} /$ fraction, 2 fractions/day $) \mathrm{D} 1$ \\
& Rest $10-12$ days \\
& 21 gy $(1.5 \mathrm{~Gy} /$ fraction, 2 fraction/day) D21 \\
& \\
& Restaging
\end{tabular}

If resectable and medically operable:

Surgery:

Midline sterno-laparotomy

Extended resection of the tumor mass

Extensive mediastinal lymph node dissection

Bilateral mediastinal lymph node dissection if $\mathrm{N} 3$

Preventive bronchial omentoplasty

Post-operative staging

Figure 1. Study design. 5 FU, 5-Fluorouracil; CDDP, cis-diaminedichloroplatinum; $D$, day.

non-small cell lung cancer (NSCLC), particularly focusing on patients with viable tumor cells after induction treatment.

\section{Patients and Methods \\ Patient Selection}

From April 1993 to June 1996, 40 patients with stage IIIB NSCLC ${ }^{8}$ diagnosed at mediastinoscopy or thoracotomy were included in the present prospective clinical trial. Potentially resectable disease after induction treatment was mandatory for inclusion. Table 1 describes the patient population selected. Further eligibility criteria included a Karnofsky index above $70 \%$, weight loss of less than $10 \%$, age below 70 years, a granulocyte and platelet count of more than $2000 / \mathrm{mm}^{3}$ and more than $100,000 / \mathrm{mm}^{3}$, respectively, a hemoglobin value of more than $10 \mathrm{~g} / \mathrm{dL}$, a serum creatinine value of less than $100 \mu \mathrm{m} / \mathrm{L}$, a predicted postoperative forced vital capacity of more than one third the value in theory, no previous malignancy (except
TABLE 1. Patient selection: Stage IIIB subgroups

\begin{tabular}{ll}
\hline $\begin{array}{l}\text { Eligible: Potentially resectable } \\
\left.\text { T4 involvements (T4 }{ }^{1}\right)\end{array}$ & \multicolumn{1}{c}{$\begin{array}{c}\text { Ineligible: Definitively } \\
\left.\text { unresectable T4 tumors (T4 }{ }^{2}\right)\end{array}$} \\
\hline $\begin{array}{l}\text { Intrapericardial pulmonary } \\
\text { artery }\end{array}$ & Malignant pleural effusion \\
$\begin{array}{l}\text { Trachea } \\
\text { Carina } \\
\text { Left atrium }\end{array}$ & $\begin{array}{l}\text { Malignant pericardial effusion } \\
\text { Diffuse mediastinal involvement* }\end{array}$ \\
Superior vena cava & $\begin{array}{l}\text { Infiltration of the heart (except } \\
\text { left atrium) }\end{array}$ \\
N3 disease & Vertebrae \\
\end{tabular}

* Mediastinal involvement that did not appear to be potentially resectable after induction therapy. 
TABLE 2. Patient characteristics

\begin{tabular}{lc}
\hline Characteristic & No. (percentage) \\
\hline Male sex & $34(85 \%)$ \\
Age $^{*}$ (y) & $55(34-69)^{*}$ \\
Weight loss $>10 \%$ & $3(7 \%)$ \\
Karnofsky index $>90 \%$ & $34(85 \%)$ \\
Histology subtype & \\
$\quad$ Adenocarcinoma & $13(32 \%)$ \\
$\quad$ Squamous cell carcinoma & $11(28 \%)$ \\
$\quad$ Large cell carcinoma & $16(40 \%)$ \\
TNM & \\
T4 N0-N2 & $21(53 \%)$ \\
T2-3 N3 & $10(25 \%)$ \\
T4 N3 & $9(22 \%)$
\end{tabular}

*Median (range).

basocellular cutaneous cancer), and no prior treatment for lung cancer. This study was approved by the ethical committee and all patients had to sign a written informed consent form before inclusion. During the same period, a total of 210 patients with stage IIIB NSCLC have been seen in our institutions.

\section{Pretreatment Evaluation}

Pretreatment evaluation included a medical history, physical examination, complete blood cell count, complete serum chemistries, electrocardiogram, ventilation-perfusion nuclide scintigraphy, and lung function tests. Tumor staging was performed by chest radiography; a computed tomographic (CT) scan of the chest, adrenal glands, liver, and brain; examination with a fiberoptic bronchoscope; and cervical mediastinoscopy or thoracotomy. Thoracoscopy, esophageal ultrasound endoscopy, angiographic CT scan, and magnetic resonance imaging were performed, if necessary, for better pretreatment staging and to assess resectability.

\section{Preoperative Chemoradiotherapy (Figure 1)}

A total dose of 42 Gy was given to the primary tumor and mediastinal lymph nodes in two sessions of accelerated hyperfractionated radiotherapy. Two doses of $1.5 \mathrm{~Gy}$ were given daily over 7 days up to a total dose of $21 \mathrm{~Gy}$ via anteroposterior fields. This was followed by a rest of 10 to 12 days. A second dose of 21 Gy was then delivered by means of the same schedule, but the first 9 Gy was delivered via anteroposterior fields, and the remaining 12 Gy was delivered via oblique fields. The target volume was delimited on the initial chest $\mathrm{x}$-ray film and CT scan of the thorax. The radiation field included the primary tumor with a $1.5-\mathrm{cm}$ margin, the ipsilateral pulmonary hilum, upper and medium mediastinal lymph nodes, and homolateral supraclavicular lymph nodes.

Two cycles of chemotherapy were to be administered on days 1 and 31; the first of the two chemotherapy cycles was delivered concomitantly with radiotherapy, but not the second cycle. Chemotherapy consisted in 5-fluorouracil, $1 \mathrm{~g} / \mathrm{m}^{2}$ in 24 hours over a 72-hour period in a continuous infusion (D1 and D31), cisplatin, $100 \mathrm{mg} / \mathrm{m}^{2}$ given 2 to 6 hours after the start of 5-fluorouracil, and vinblastine, 4 $\mathrm{mg} / \mathrm{m}^{2}$. The second chemotherapy course on day 31 was postponed if patients had a granulocyte count of less than $1500 / \mathrm{mm}^{3}$ or a platelet count of less than $100,000 / \mathrm{mm}^{3}$ and until these values were reached.
TABLE 3. Results of induction chemoradiotherapy in 40 patients:

\begin{tabular}{lrcr}
\hline Results & No. & \% of overall & $\begin{array}{c}\text { \% of } \\
\text { evaluated }\end{array}$ \\
\hline Mediastinitis & 1 & 2 & \\
Early deaths & 3 & 6 & \\
$\quad$ Treatment related & 1 & 2 & \\
$\quad$ Progression & 2 & 4 & \\
Eligible for evaluation on day 65 & 36 & 92 & 100 \\
$\quad$ Progressive disease & 6 & 12 & 16 \\
Stable disease & 1 & 2 & 3 \\
Partial response & 27 & 68 & 75 \\
$\quad$ Complete response & 2 & 4 & 6 \\
Eligible for surgery & 29 & 73 & 80 \\
\hline
\end{tabular}

\section{Response and Toxicity of Chemoradiotherapy}

Response to induction treatment was assessed by means of the standard World Health Organization (WHO) criteria. Complete response to induction treatment was defined as the complete eradication of tumor. Partial response was defined as a 50\% reduction in the sum of the products of all measurable lesions or a decrease $(50 \%)$ in the size of assessable but nonmeasurable lesions. Stable disease was defined as a change in the sum of the products of all measurable lesions, between less than a $50 \%$ reduction and less than $25 \%$ progression. Definitive progressive disease was defined as a $25 \%$ increase in the sum of the products of tumor parameters or the appearance of new tumor lesions. Toxicity induced by preoperative treatment was assessed by means of WHO criteria.

\section{Postchemoradiotherapy Staging}

At day 65, restaging was done in all patients with a chest x-ray film, CT scan of the thorax, the upper part of the abdomen, and brain, and examination with a fiberoptic bronchoscope. This new staging procedure was performed to assess the clinical tumor response and to select patients for surgery. Lung function tests and ventilationperfusion nuclide scintiscans were performed to assess whether examination results did not contraindicate surgery.

\section{Surgery (Figure 1)}

All patients treated with induction chemoradiotherapy and having a clinical response were selected for surgery. The surgical approach was a midline sternolaparotomy in most of the cases. When the lower part of the trachea or the carina was involved at the first staging procedure, the surgical approach was a right thoracotomy. A right thoracotomy associated with a laparotomy or a sternolaparotomy was the surgical option in cases of vena caval involvement. For left-sided tumors, particularly from the left lower lobe, or when a great vessel was involved on the left side (pulmonary artery), the surgical approach was a left thoracotomy in association with either a laparotomy or a sternolaparotomy, according to the nodal status (N2 or N3). The objective of surgery was to completely remove all tumor tissue. Lymph node dissection consisted in the resection of all ipsilateral mediastinal lymph node stations in all cases. In case of N3 disease, a bilateral mediastinal lymph node dissection was performed. After lung resection, the 
bronchial stump was wrapped in a pedicled omental flap in all cases, to avoid bronchial complications resulting from preoperative chemoradiotherapy and particularly stump insufficiency.

After surgery and according to pathologic findings, patients were classified as having had a complete or an incomplete response to induction treatment and a complete or a partial resection. The status of mediastinal lymph nodes after induction treatment was also recorded.

\section{Follow-up}

All patients were monitored every 3 months for the first 2 years from the end of the treatment and every 6 months during the next 3 years. Tests conducted during follow-up visits included a physical examination, a complete blood cell count and serum chemistry assessment, and a chest x-ray film. A CT scan of the thorax was obtained every 6 months after the end of the treatment, and examination with a fiberoptic bronchoscope was performed every year after the end of the treatment.

\section{Statistical Analysis}

Survival was measured from the first day of radiotherapy until death or the last follow-up visit. The postoperative death and morbidity rates are defined as events occurring before hospital discharge. The locoregional control rate is defined as the percentage of patients free of tumor in the ipsilateral lung, the mediastinum, or the supraclavicular area. This definition is derived from that proposed by Arriagada and associates. ${ }^{4}$ Relapse-free survival was the time from radiotherapy until disease progression. Survival curves were evaluated by the Kaplan-Meier method. ${ }^{9}$ Median follow-up was calculated by the method of Schemper and Smith. ${ }^{10}$ Multivariate analysis of prognostic factors for survival and relapse was performed by the Cox model. Multivariate analysis included 4 parameters (10\% of the number of patients): pretreatment $\mathrm{T}$, pretreatment $\mathrm{N}$, postinduction $\mathrm{N}$, and postinduction $\mathrm{T}$.

\section{Results}

\section{Patient Characteristics}

The present analysis was conducted on April 1, 2000, with updated data. Median follow-up is 64 months and the minimum follow-up for survivors is 48 months. No patient has been lost to follow-up. Patient characteristics are summarized in Table 2. The Karnofsky index was more than $90 \%$ in $85 \%$ of patients. The histologic type was adenocarcinoma in $13(32 \%)$ patients, squamous cell carcinoma in $11(28 \%)$ patients, and large cell carcinoma in $16(40 \%)$ patients. A brain, upper abdominal, and chest CT scan, a liver ultrasound, and a bone scan were performed on all patients. Surgical thoracic staging (mediastinoscopy or thoracotomy) was performed on all patients. Thirty patients had a T4 tumor based on evidence obtained at radiologic and surgical staging. Among these 30 patients, 5 had a T4 N0, 1 a T4 N1, 15 a T4 N2, and 9 a T4 N3 tumor. Nineteen patients had N3 involvement at diagnosis, and 1 of them had supraclavicular lymph node involvement. Proof of N2 and N3 disease was based on the histologic analysis of the biopsy specimen obtained at mediastinoscopy.

\section{Induction Chemoradiotherapy (Table 3)}

One patient did not receive the planned doses of chemoradiotherapy because of coagulase-positive staphylococcusinduced mediastinitis. Three deaths occurred, 2 due to severe hemoptysis related to locoregional progression (days 46 and 57) and 1 due to grade 4 neutropenia and thrombocytopenia (day 43). Four patients were therefore not evaluated on day 65 . No grade $3 / 4$ esophageal toxicity was noted. Six patients had grade $3 / 4$ neutropenia.

A clinical tumor response was obtained in 29 patients (73\%) during chemoradiotherapy (2 clinical complete responses). Disease was stable in 1 patient but progressed in 6 patients. The 29 (73\%) patients who had a clinical tumor response after induction treatment were therefore eligible for surgery. Patients who did not have a tumor response (n $=7)$ or who had mediastinitis $(n=1)$ were not eligible for surgery.

\section{Surgery}

The median time from the first day of chemoradiotherapy to surgery was 77 days (range 63-90 days). Twenty-nine patients underwent a thoracotomy, which revealed unresectable disease in 5 patients. Tumor resection was therefore feasible in $24(60 \%)$ patients. A pneumonectomy was performed on 18 patients and a lobectomy on 6 . Twenty-one patients, including all patients with $\mathrm{N} 3$ disease at initial staging, underwent a bilateral mediastinal lymphadenectomy and 3 an ipsilateral mediastinal lymph node dissection. The postchemoradiotherapy mediastinal lymph node status was therefore assessable in all patients treated with surgery. The vena cava was resected in 4 patients, a great vessel in 3 patients, the left atrium in 7 patients, the trachea in 3 patients, and the carina in 3. In 18 patients treated with pneumonectomy, the bronchial stump was wrapped in a pedicled omental flap. The postoperative death rate was $7 \%$ (2 patients). Postoperative morbidity affected 7 patients $(24 \%)$ and included acute respiratory distress syndrome $(\mathrm{n}=2)$, phrenic nerve paralysis $(\mathrm{n}=$ $1)$, prolonged assisted ventilatory support $(n=1)$, cardiac failure during surgery $(n=1)$, persistent pleural effusion $(n=1)$, and omental flap necrosis $(n=1)$. No stump insufficiency nor morbidity related to laparotomy was noted. The median duration of the postoperative hospital stay was 20 days.

\section{Pathologic Findings}

A histologic complete resection was achieved in $23(58 \%)$ patients. A histologic complete response was obtained in 4 (10\%) patients. Ten patients with N2/N3 disease (30\%) had a complete disappearance of mediastinal lymph node involvement (mediastinal lymph node down staging) after chemoradiotherapy. Twenty-five patients who had a clinical objective tumor response and who had persistent viable tumor cells in the thorax were submitted to surgery. These 25 patients were defined as partial responders. Thirteen partial responders 


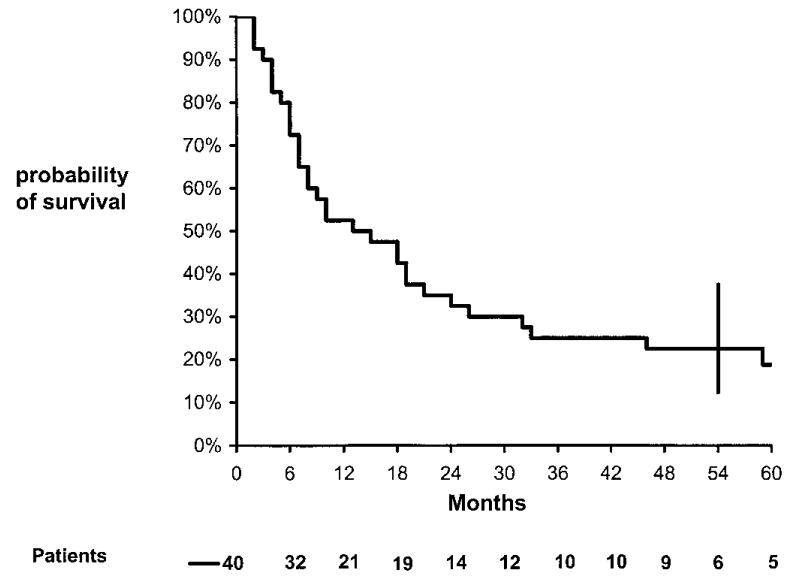

Figure 2. Survival of the overall population.

TABLE 4. Multivariate analysis for survival and relapse

\begin{tabular}{lll}
\hline & \multicolumn{2}{c}{ Relative risk } \\
\cline { 2 - 3 } & \multicolumn{1}{c}{ Survival } & \multicolumn{1}{c}{ Relapse } \\
\hline Pretreatment N & $1.2(P=.7)$ & $1.3(P=.6)$ \\
Pretreatment T & $1.3(P=.5)$ & $1.3(P=.6)$ \\
Postinduction N & $3.1(P=.005)$ & $4.9(P=.001)$ \\
Postinduction T & $2.1(P=.06)$ & $2.0(P=.08)$ \\
\hline
\end{tabular}

were classified as postinduction N0/N1. Resection was complete in 20 of these partial responders.

\section{Survival Analysis}

Of the 40 patients, $7(17.5 \%)$ were alive at $48,53,54,67,72$, and 73 months, and $5(12.5 \%)$ of them were free of disease at $48,53,67,72$, and 73 months. All these 7 patients had persistent viable tumor cells after induction treatment and 6 of them had no mediastinal lymph node involvement at the time of surgery (postinduction N0/N1).

In 8 patients the disease progressed during chemoradiotherapy. Progression occurred in the primary site $(\mathrm{n}=5)$, bone $(\mathrm{n}=3)$, brain $(\mathrm{n}=2)$, and liver $(\mathrm{n}=1)$. Of the 29 patients who underwent thoracotomy, 19 (65\%) have had a relapse. Thirteen (45\%) had a distant metastasis as the first relapse event. The first site of distant metastasis was the contralateral lung $(\mathrm{n}=5)$, liver $(\mathrm{n}=1)$, bone $(\mathrm{n}=3)$, brain $(\mathrm{n}=6)$, distant lymph node $(\mathrm{n}=1)$, or another site $(\mathrm{n}=2)$. Five patients had multiple sites of metastasis. Seven (24\%) patients had a locoregional relapse after surgery. Five (20\%) partial responders had a locoregional relapse. When overall patients are considered, 12 (30\%) have had a locoregional relapse. Twenty-three percent of the overall population (95\% confidence interval [CI]: $11 \%-41 \%$ ) had not had a relapse at 5

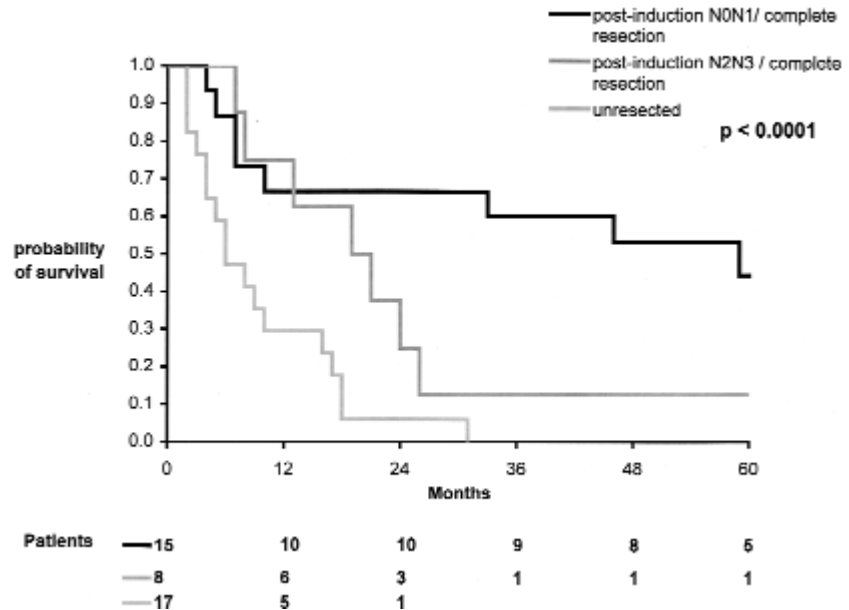

Figure 3. Survival according to postinduction lymph node status and resection.

years. When only partial responders were considered $(\mathrm{n}=25)$, $22 \%$ (95\% CI: $10 \%-34 \%$ ) had not had a relapse at 5 years.

Thirty-three deaths occurred. Of these, 8 (24\%) occurred in patients with no evidence of recurrent disease. These 8 patients died of thrombocytopenia and neutropenia $(n=1)$, myocardial infarction $(n=2)$, and lung insufficiency $(n=5)$. Lung insufficiency was related to acute respiratory distress syndrome $(\mathrm{n}=3)$, pulmonary embolism plus interstitial pneumonitis $(\mathrm{n}=1)$, and postradiation pneumonitis $(\mathrm{n}=1)$. Of these, $4(10 \%)$ were directly related to treatment.

The 5-year overall survival for the 40 patients is 19\% (CI 95\%: 10\%-34\%) (Figure 2). The 5-year overall survival is $23 \%(11 \%-40 \%)$ and $17 \%$ (6\%-39\%) for patients with T4 NX and TX N3, respectively. The 5-year overall survival is $42 \%$ for postinduction $\mathrm{N} 0 / \mathrm{N} 1$ treated with complete resection, $12 \%$ for postinduction N2/N3 treated with complete resection, and $0 \%$ for patients who were not treated with complete resection $(P<.001)$ (Figure 3). A multivariate analysis (Table 4) showed that postinduction mediastinal lymph node status was the only prognostic factor for survival and relapse (relative risk for death, 3.1; relative risk for relapse, 4.9; $P=.005$ and .001, respectively).

The 4 patients who had a complete response after chemoradiotherapy died after 5, 6, 58, and 60 months. None of these patients had a relapse. The 5-year survival is $28 \%$ (CI 95\%: 14\%-47\%) for the partial responders (Figure 4) and 35\% for those treated with complete resection $(\mathrm{n}=20)$. The 5-year survival is $47 \%$ for partial responders with postinduction N0/N1. Two of the 11 patients treated with superior vena caval resection or a left atrial resection survived 5 years.

\section{Discussion}

This study shows that surgery can achieve a promising $28 \%$ long-term survival in patients with stage IIIB NSCLC who only partially responded to chemoradiotherapy (partial responders). 
TABLE 5. Review of resections and survivals of patients with stage IIIB cancer treated with postinduction surgery

\begin{tabular}{lllll}
\hline & SWOG $^{\mathbf{5}}$ & WGCC $^{\mathbf{6}}$ & GLCCG $^{\mathbf{7}}$ & \multicolumn{1}{c}{ CEBI } \\
\hline No. of cases & 51 & 42 & 29 & 40 \\
Clinical response rate & $59 \% *$ & $64 \% *$ & $62 \%$ & $73 \%$ \\
Resection rate & $24 \%$ & $45 \%$ & $68 \%$ & $58 \%$ \\
Survival & $24 \%(3 \mathrm{y})$ & $26 \%(4 \mathrm{y})$ & $26 \%(3 \mathrm{y})$ & $19 \%(5 \mathrm{y})$ \\
\hline
\end{tabular}

SWOG, Southwest Oncology Group; WGCC, West German Cancer Center; GLCCG, German Lung Cancer Cooperative Group; CEBI, current study.

*Including stages IIIA and IIIB.

Chemoradiotherapy is considered the standard treatment for stage IIIB NSCLC. ${ }^{1-3}$ Nevertheless, the locoregional control rate, defined as the percentage of patients free of residual tumor in the thorax after treatment, is a modest $17 \%$ at 1 year. ${ }^{4}$ The persistence of viable tumor cells in the primary tumor site or in the mediastinum after chemoradiotherapy is a valid marker of treatment failure and death, whatever the clinical response. ${ }^{11}$ This was demonstrated in a series of 63 patients with stage III disease treated with chemoradiotherapy who had persistent viable tumor cells at bronchoscopy. In this prospective study, the 3-year operative survival was only $3 \%$ for these patients. Because locoregional control is determinant in the outcome of stage III disease, it was the rationale behind the inclusion of surgery in a multimodality treatment approach. In this setting, postinduction surgery is considered a salvage procedure aimed at achieving locoregional control. Previous studies of multimodality treatment reported 3-year operative survivals ranging from $24 \%$ to $26 \%$, complete resection rates from $50 \%$ to $70 \%$, and locoregional failure rates of only $20 \%$ to $38 \% .^{5-7}$ Our results are in the same range, with a 3-year operative survival of $28 \%$, a $58 \%$ complete resection rate, and a $30 \%$ locoregional failure rate. All together, these results are encouraging when compared with the large phase III chemoradiotherapy studies conducted by Le Chevalier, ${ }^{2}$ Sause, ${ }^{3}$ and their coworkers, but not convincing when compared with phase III trials of chemoradiotherapy performed in well-selected populations. ${ }^{12,13}$

Because the persistence of viable tumor cells after chemoradiotherapy is considered a marker of treatment failure, we looked at the effect of surgery in patients who only partially responded to chemoradiotherapy. Surprisingly, surgery successfully salvaged a population rarely considered curable and obtained an interesting $28 \%$ long-term survival. A comparable result was achieved in the Southwest Oncology Group (SWOG) study, ${ }^{5}$ which reported a similar outcome for patients with a pathologic complete response after chemoradiotherapy and those with persistent viable tumor after chemoradiotherapy and treated by surgery. In the German study, ${ }^{6}$ the 4 -year survival was $46 \%$ for patients treated with surgery and having persistent viable tumor cells after chemoradiotherapy. These data suggest that surgery is an effi-

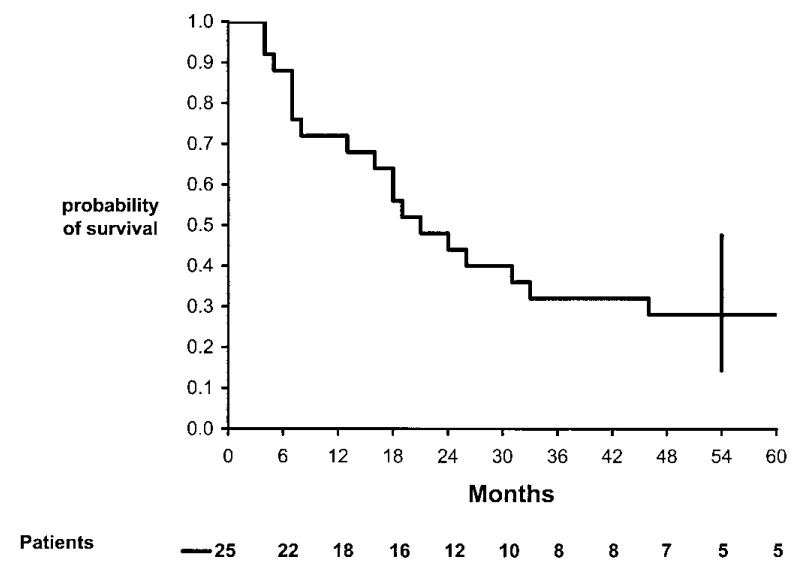

Figure 4. Survival of partial responders.

cient salvage treatment for patients with stage IIIB NSCLC with viable residual disease after chemoradiotherapy alone. On the other hand, none of the 4 patients who had a histologic complete response after induction treatment are alive. The rationale for surgery is questionable in these patients in whom preoperative evaluation of a pathologic complete response, with the use of new staging techniques such as positron emission tomographic scanning, is probably required.

Previous studies $5,6,13$ have reported that the mediastinal lymph node status after induction treatment had a prognostic significance for patients treated with postinduction surgery. In a study including 42 patients with stage IIIAN2 disease treated with multimodality therapy, Choi and colleagues ${ }^{14}$ reported that the degree of lymph node down staging was translated into a survival benefit since the 5-year survivals were $79 \%, 42 \%$, and $18 \%$ for postoperative tumor stages $0 / \mathrm{I}$, II, and III, respectively. In the SWOG study, ${ }^{5}$ the strongest predictor of long-term survival after thoracotomy was the absence of tumor in the mediastinal nodes (3-year survival $44 \%$ vs $18 \% ; P=.0005)$. In the German study, 4-year survivals were $38 \%$ and $15 \%(P=.11)$ for postinduction N0/N1 and N2/N3 patients, respectively. Multivariate analysis performed in our study fully confirms these data since it shows that the presence of mediastinal lymph node involvement after induction treatment is the most relevant prognostic indicator. Altogether these studies suggest that the postinduction $\mathrm{N} 2 / \mathrm{N} 3$ status is a marker of microscopic metastatic disease and that surgery is not associated with a significant longterm survival in these patients. On the other hand, the absence of mediastinal lymph node involvement after induction therapy for patients with stage III disease is always associated with a long-term survival of more than $30 \%$ after surgery, whatever the initial $\mathrm{T}$ stage.

Specific procedures such as bilateral lymphadenectomy, extrapulmonary resections, and median sternotomy are required for postinduction surgery for patients with stage 
IIIB NSCLC. Initial reports ${ }^{14}$ documented high rates of postoperative complications (46\%) and mortality (8.6\%) in patients treated with preoperative chemoradiotherapy. In their respective studies, Albain, ${ }^{5}$ Eberhardt, ${ }^{6}$ and their associates reported high rates of non-cancer-related deaths (25\% and $26 \%$ of the overall population). Our study confirms this finding inasmuch as $8(20 \%)$ deaths occurred in patients in complete remission. Five of these deaths were due to lung insufficiency and 2 to myocardial infarction. Our study and others reported a higher rate of acute respiratory distress syndrome or pneumonitis after multimodality treatment compared with standard lung resection alone. These data indicate that patient management needs to be improved to decrease toxicities. In particular, radiotherapy schedules, surgical procedures, and postoperative care (intensive care and long-term follow-up) need to be reappraised and redefined to avoid these complications. Previous studies suggested that preoperative chemotherapy and/or radiotherapy could increase the rate of bronchial stump insufficiency. ${ }^{15}$ Some teams ${ }^{16,17}$ perform omentoplasty to avoid this complication. In the present study, the bronchial stump was wrapped in a pedicled omental flap in 18 cases. No stump insufficiency was noted with this procedure, which suggests that it is an efficient way of avoiding stump insufficiency.

Stage IIIB NSCLCs are classified as "unresectable locally advanced NSCLC" in the current TNM staging system. ${ }^{18}$ Nevertheless, our study and previous studies (Table 5) show that surgery makes it possible both to achieve a high rate of complete remission and to salvage patients with residual disease after chemoradiotherapy. This argues for a change in the TNM classification. In a recently published proposition, ${ }^{19,20}$ selected patients with stage IIIB disease and clinically detectable N2 disease were classified in a subgroup designated "potentially resectable." Further studies are needed to confirm the relevance of this new subclassification.

In conclusion, this study suggests that postchemoradiotherapy surgery is an efficient salvage procedure for selected patients with stage IIIB disease who are partial responders without mediastinal lymph node involvement at the time of surgery. Altogether these data suggest that postinduction TN status is more accurate than pretreatment TN status to guide the indications for surgery for patients with stage IIIB disease. This study raises several questions: What is the best preoperative strategy to increase the rate of mediastinal lymph node down staging? How can toxicity induced by multimodality treatment be lessened? Faced with such toxicity, should surgery be performed in patients having a complete response,and how can they be identified preoperatively?

We thank Lorna Saint-Ange for editing.

\section{References}

1. Dillman RO, Seagren SL, Propert KJ, Guerra J, Eaton WL, Perry MC, et al. A randomized trial of induction chemotherapy plus high-dose radiation versus radiation alone in stage III non-small cell lung cancer. N Engl J Med. 1990;323:940-5.

2. Le Chevalier T, Arriagada R, Tarayre M, Lacombe-Terrier MJ, Laplanche A, Quoix E, et al. Significant effect of adjuvant chemotherapy on survival in locally advanced unresectable non-small cell lung carcinoma. J Natl Cancer Inst. 1992;84:58.

3. Sause W, Kolesar P, Taylor S 4th, Johnson D, Livingston R, Komaki R, et al. Final results of phase III trial in regionally advanced unresectable non-small cell lung cancer: Radiation Therapy Oncology Group and Eastern Cooperative Oncology Group, and Southwest Oncology Group. Chest. 2000;117:358-64.

4. Arriagada R, Le Chevalier T, Quoix E, Ruffie P, de Cremoux H, Douillard JY, et al. ASTRO plenary: effect of chemotherapy on locally advanced non-small cell lung carcinoma: a randomized study of 353 patients. GETCB, FLNCC and the CEBI trialists. Int J Radiat Oncol Biol Physiol. 1991;20:1183-90.

5. Albain KS, Rusch VW, Crowley JJ, Rice TW, Turrisi AT 3rd, Weick $\mathrm{JK}$, et al. Concurrent cisplatin/Etoposide plus chest radiotherapy followed by surgery for stages IIIA (N2) and IIIB non-small cell lung cancer: mature results of Southwest Oncology Group phase II study 88-05. J Clin Oncol. 1995;13:1880-92.

6. Eberhardt W, Wilke H, Stamatis G, Stuschke M, Harstrick A, Menker $\mathrm{H}$, et al. Preoperative chemotherapy followed by concurrent chemoradiation therapy based on hyperfractionated accelerated radiotherapy and definitive surgery in locally advanced non-small cell lung cancer: mature results of a phase II trial. J Clin Oncol. 1998;16:622-34.

7. Thomas M, Rube C, Semik M, von Eiff M, Freitag L, Macha HN, et al Impact of preoperative bimodality induction including twice daily radiation on tumor regression and survival in stage III non-small cell lung cancer. J Clin Oncol. 1999;17:1187.

8. Mountain CF. A new international staging system for lung cancer. Chest. 1986;89:225-33.

9. Kaplan EL, Meier P. Nonparametric estimation from incomplete observations. J Am Stat Assoc. 1958;53:457-81.

10. Schemper M, Smith T. A note on quantifying follow-up in studies of failure time. Controlled Clin Trials. 1996;17:343-6.

11. Andre F, Grunewald D, Le Chevalier T. Persistence of viable tumor cells after radiation and chemotherapy for stage IIIB non-small cell lung cancer: an early marker of treatment failure. J Thorac Cardiovasc Surg. 2001;121:403.

12. Dillman RO, Herndon J, Seagren SL, Eaton WL Jr, Green MR Improved survival in stage III non small cell lung cancer: seven year follow-up of cancer and leukemia group B (CALGB) 8433 trial. J Natl Cancer Inst. 1996;88:1210-5.

13. Furuse K, Fukuoka M, Kawahara M, Nishikawa H, Takada Y, Kudoh $S$, et al. Phase III study of concurrent versus sequential thoracic radiotherapy in combination with mitomycin, vindesine, and cisplatin in unresectable stage III non-small cell lung cancer. J Clin Oncol. 1999; 17:2692-9.

14. Choi NC, Carey RW, Daly W, Mathisen D, Wain J, Wright C, et al. Potential impact on survival of improved tumor down staging and resection rate by preoperative twice-daily radiation and concurrent chemotherapy in stage IIIA non-small cell lung cancer. J Clin Oncol. 1977; 15:712-22.

15. Hubaut JJ, Baron O, Al Habash O, Despins P, Duveau D, Michaud JL. Closure of the bronchial stump by manual suture and incidence of bronchopleural fistula in a series of 209 pneumonectomies for lung cancer. Eur J Cardiothorac Surg. 1999;16:418-23.

16. Akopov AL, Mosin IV. The possibilities of greater omentum usage in thoracic surgery. Eur J Cardiothorac Surg. 1999;15:465-8.

17. Grunenwald D, Baldeyrou P, Dennewald G, Girard P, Rogier M. Preventive bronchial omentoplasty and myoplasty in pulmonary resection: technical aspects and results. Ann Chir. 1994;48 248-51.

18. Mountain CF. Revisions in the international system for staging lung cancer. Chest. 1997;111:1711-7.

19. Grunenwald D, Le Chevalier T. Stage IIIA category of non-small cell lung cancer: a new proposal. J Natl Cancer Inst. 1997;89:88-9.

20. Grunenwald DH. Surgery for advanced stage lung cancer. Semin Surg Oncol. 2000;18:137-42. 